\title{
Sunflower Meal and Enzyme Supplementation of The Diet of 21- to 42-d-old Broilers
}

\section{-Author(s)}

Araújo WAG de!

Albino LFT"

Rostagno HS"

Hannas MI"

Pessoa GBS"II

Messias RKGII

Lelis GR"II

Ribeiro Jr VIII

Professor IFNMG. E.mail: aziszoo@yahoo com.br

Professor DZO/UFV

III Ph.D, student, DZO/ UFV.

Part of the Ph.D. thesis presented to DZO/UFV by the first author. Project funded by FAPEMIG.

\section{Mail Adress}

Corresponding author e-mail address

E-mail: aziszoo@yahoo

\section{घKeywords}

Sunflower meal, broilers, enzyme blend, and alternative feedstuffs.

\section{ABSTRACT}

The objective of this study was to evaluate the performance of male broilers between 21 and 42 days of age fed diets supplemented with an enzyme blend (EB) and containing graded levels of sunflower meal (SFM). In total, 1920 male Cobb broilers were distributed according to a randomized block experimental design in a $4 \times 3$ factorial arrangement (four SFM dietary levels vs. three feed formulations) with eight replicates of 20 birds each. Dietary SFM inclusion levels were 0, 8, 16, and 24\%. Feed formulation included one negative control diet (NC) formulated to supply broiler nutritional requirements, considering EB nutritional matrix values; a diet formulated as the first one, but with the addition of $0.5 \%$ $\mathrm{EB}(\mathrm{NC}+\mathrm{EB})$; and the third diet (positive control - PC) was calculated to supply broiler requirements. Feed intake, weight gain, feed conversion ratio, and carcass parameters were evaluated. There was no effect of EB dietary supplementation on the evaluated performance or carcass parameters $(p>0.05)$. Increasing dietary addition of SFM reduced weight gain and worsened feed conversion ratio ( $p>0.05)$. The best EEI was obtained with the NC+EB diet containing 8.0\% SFM.

\section{INTRODUCTION}

Brazilian production of sunflower seeds account for only $0.003 \%$ of global production, but it is rapidly expanding. In 2009, total sunflower crop area was 87,800 ha, with a productivity of $1463 \mathrm{~kg} / \mathrm{ha}$, resulting a total production of 128,500 tons of sunflower (Rosa et al., 2009).

In Brazil, sunflower is mostly used for oil production. In addition of producing edible oil, sunflower is also a potential renewable energy source, as it can be used as a raw material for biodiesel (Porto et al., 2008). The global demand for sunflower oil has increased $1.8 \%$ per year, in average, whereas the domestic demand grows 13\% per annum (Rosa et al., 2009), resulting in a gradual increase in sunflower crop area in Brazil.

Sunflower meal (SFM) is a by-product of the sunflower oil industry, and has been increasingly added to feeds, particularly in the south and mid-west of Brazil. The main challenge of including SFM in broiler feeds is its high fiber content (NRC 1994, FEDNA 2003, INRA 2004), which may negatively influence performance and carcass quality. Some exogenous enzyme may be added to broiler diets containing SFM to aid fiber digestion (carbohydrases) or to solubilize phytic phosphorus (phytase), thereby reducing their negatives effects on broiler production parameters.

Reports on the results of the inclusion of sunflower meal in broiler feeds are controversial. According to Furlan et al. (2001), sunflower meal, in replacement of soybean protein, can be added up to $30.0 \%$ (13.17 and $12.04 \%$ for feed intake and weight gain, respectively), 
with no detrimental effects on performance. Senkoylu et al. (2006) did not observe any effect on broiler performance when up to $28 \%$ SFM was included in the feed. However, those authors used the residue SFM cold-pressing, which is more nutritious $(32.3 \%$ CP and $18.78 \%$ EE) than sunflower meal. Tavernari et al. (2009) did not find weight gain differences in broilers fed up to $20.0 \%$ SFM.

On the other hand, Pinheiro et al. (2002) found that SFM levels higher than $12.0 \%$ reduced broiler weight gain, as well as feed intake, while obtaining the best feed intake when SFM was not added to the feed. Tavernari et al. (2009) also reported that broiler feed intake was inversely proportional to SFM dietary level.

Exogenous enzymes are frequently added to feeds to improve the nutritional value of feedstuffs, particularly of those containing high fiber levels (Kocher et al., 2000). Tavernari et al. obtained better dry matter digestibility and better coefficients of $\mathrm{Ca}$ and $\mathrm{P}$ metabolizability in broilers fed diets with SFM supplemented with enzymes. Kocher et al. (2000), on the other hand, did not observe any effects of enzyme addition in feeds containing sunflower meal.

High sunflower meal levels in broiler diets require the addition of high oil levels in order to compensate the low energy content of SFM. Oil is one of the most expensive feedstuffs in broiler diets.

The objective of this study was to evaluate the performance, carcass traits and economics of the inclusion of different sunflower meal levels in diets supplemented or not with an enzyme complex fed to 21- to 42-d-old broilers.

\section{MATERIAL AND METHODS}

The experiment was carried out between May and June, 2010, at the facilities of the poultry sector of the Department of Animal Science of Universidade Federal de Viçosa, MG, Brazil.

In the study, 1920 male Cobb broilers, with 0.852 $\pm 0.012 \mathrm{~kg}$ average initial weight were evaluated in the period of 21 to 42 days of age. Birds were housed in a masonry broiler house, with open-sides with wire mesh, and clay tiles subdivided in $1.0 \times 1.5 \mathrm{~m}$ pens covered with wood-shavings litter and equipped with nipple drinkers and tube feeders. House temperature was measured once daily $(08: 00 \mathrm{~h})$ to determine maximum and minimum temperatures during the experimental period.

Broilers were distributed according to a randomized block experimental design in a $4 \times 3$ factorial arrangement (four SFM dietary levels vs. three feed formula- tions) with eight replicates of 20 birds each. Pens were arranged in four longitudinal lines in the house, each with the same number of experimental units (pens), and considered as one block.

Sunflower meal was included at 0, 8, 16, and 24\% of the diets with three different formulations (Table 1). The positive control (PC) diets were calculated to supply broilers' nutritional requirements according to Rostagno etal. (2005; Table 2). The negative control (NC) diets were calculated considering the nutritional matrix of the enzyme blend (EB) but were not supplemented with $\mathrm{EB}$. The $\mathrm{NC}+\mathrm{EB}$ diets were calculated as $\mathrm{NC}$ and supplemented with $0.005 \%$ EB. The enzyme blend, containing carbohydrases and phytase was added to improve the digestibility of the feed components that serve as substrate for these enzymes.

The chemical composition of sunflower meal (Table 3) was determined at the feedstuff analysis laboratory of the Department of Animal Science of the Federal University of Viçosa, and its digestible amino acids were taken from literature (NRC, 1994; FEDNA, 2003; INRA, 2004; Tavernari et al., 2010).

Mortality was recorded to allow performance data correction. Birds and feeds were weighed in the beginning and at the end of the experimental period ( 21 and 42 days) to calculate weight gain, feed intake, feed conversion ratio, livability, and production efficiency index (PEI) of 42-d-old birds, according to the equations: Livability $=100-\mathrm{MO}$, and $\mathrm{PEI}=[(\mathrm{ABW}$ * livability)/MA * FCR]*100, where $\mathrm{MO}=$ mortality, $A B W$ $=$ average body weight at slaughter; $\mathrm{MA}=$ market age, and FCR = feed conversion ratio.

In order to evaluate the economic viability of the inclusion of sunflower meal in the diet, diet cost was determined in Brazilian real $(R \$)$, per $\mathrm{kg}$ live weight produced (Yi). The following equation, adapted from Bellaver et al. (1985) was applied: $\mathrm{Yi}=(\mathrm{Pi}$ * Qi) / Ei, where $\mathrm{Yi}=$ cost of the kilogram of live weight in the i-th treatment (sunflower meal level); $\mathrm{Pi}=$ price per kilogram of the diet utilized in the i-th treatment; Qi, quantity of diet consumed in the i-th treatment; and $\mathrm{Ei}=$ kilogram of live weight produced. The economic efficiency index (EEI) was then calculated as: $E E I=(L C e$ / CTei) * 100, in which $\mathrm{LCe}=$ lowest cost of diet per live weight produced observed among treatments; and $\mathrm{CTEi}=$ cost of the i-th treatment.

The following price/kg of the ingredients used to calculate the costs were obtained in the city of Viçosa in May, 2010: antioxidant ( $R \$$ 6.82), limestone ( $R \$$ 0.028), choline chloride $(R \$ 2.92)$, enzyme complex $(R \$ 6.43)$, DL-methionine ( $R \$ 8.15)$, soybean meal ( $R \$$ $0.63)$, sunflower meal $(R \$ 0.31)$, dicalcium phosphate 
( $R \$$ 1.18), L-lysine ( $R \$ 4484)$, L-threonine ( $R \$ 8.15)$, corn ( $R \$ 0.53)$, oil $(R \$ 2.57)$, salt $(R \$ 0.19)$, salinomycin $(R \$ 2.195)$, vitamin supplement $(R \$ 3.30)$ and mineral supplement (R\$1.86).
Three birds per replicate were sacrificed at 42 days of age to determine carcass, breast, breast fillet, thighs and drumstick, and abdominal fat yields relative to cold carcass weight (after the chiller).

Table 1 - Ingredients and chemical composition of broiler feeds containing different sunflower meal levels and supplemented or not with an enzyme blend, on as-fed basis.

\begin{tabular}{|c|c|c|c|c|c|c|c|c|c|c|c|c|}
\hline \multirow[b]{2}{*}{ Ingredients (\%) } & \multicolumn{4}{|c|}{ NC } & \multicolumn{4}{|c|}{$\mathrm{NC}+\mathrm{EB}$} & \multicolumn{4}{|c|}{$P C$} \\
\hline & $0 \%$ & $8 \%$ & $16 \%$ & $24 \%$ & $0 \%$ & $8 \%$ & $16 \%$ & $24 \%$ & $0 \%$ & $8 \%$ & $16 \%$ & $24 \%$ \\
\hline Corn & 66.823 & 61.016 & 55.208 & 49.401 & 66.812 & 61.005 & 55.198 & 49.391 & 63.245 & 57.438 & 51.631 & 45.824 \\
\hline Soybean meal & 28.523 & 25.046 & 21.568 & 18.09 & 28.525 & 25.047 & 21.57 & 18.092 & 29.954 & 26.477 & 22.999 & 19.521 \\
\hline Sunflower meal & 0.000 & 8.000 & 16.000 & 24.000 & 0.000 & 8.000 & 16.000 & 24.000 & 0.000 & 8.000 & 16.000 & 24.000 \\
\hline Soybean oil & 1.280 & 2.577 & 3.874 & 5.171 & 1.284 & 2.581 & 3.878 & 5.174 & 3.027 & 4.324 & 5.621 & 6.918 \\
\hline Dicalcium phosphate & 1.109 & 1.108 & 1.106 & 1.104 & 1.109 & 1.108 & 1.106 & 1.104 & 1.651 & 1.650 & 1.648 & 1.646 \\
\hline Limestone & 0.989 & 0.952 & 0.914 & 0.877 & 0.989 & 0.952 & 0.914 & 0.877 & 0.845 & 0.808 & 0.771 & 0.733 \\
\hline Salt & 0.476 & 0.440 & 0.405 & 0.369 & 0.476 & 0.440 & 0.405 & 0.369 & 0.477 & 0.441 & 0.406 & 0.370 \\
\hline DL-Methionine 99\% & 0.198 & 0.187 & 0.176 & 0.164 & 0.198 & 0.187 & 0.176 & 0.164 & 0.208 & 0.197 & 0.186 & 0.175 \\
\hline L-Lysine HCL 99\% & 0.207 & 0.272 & 0.336 & 0.400 & 0.207 & 0.272 & 0.336 & 0.400 & 0.196 & 0.260 & 0.324 & 0.388 \\
\hline L-threonine $98 \%$ & 0.075 & 0.084 & 0.093 & 0.103 & 0.075 & 0.084 & 0.093 & 0.103 & 0.076 & 0.085 & 0.094 & 0.104 \\
\hline Vitamin premix $^{1}$ & 0.100 & 0.100 & 0.100 & 0.100 & 0.100 & 0.100 & 0.100 & 0.100 & 0.100 & 0.100 & 0.100 & 0.100 \\
\hline Mineral premix ${ }^{2}$ & 0.050 & 0.050 & 0.050 & 0.050 & 0.050 & 0.050 & 0.050 & 0.050 & 0.050 & 0.050 & 0.050 & 0.050 \\
\hline Choline chloride, $60 \%$ & 0.100 & 0.100 & 0.100 & 0.100 & 0.100 & 0.100 & 0.100 & 0.100 & 0.100 & 0.100 & 0.100 & 0.100 \\
\hline Salinomycin, $12 \%$ & 0.060 & 0.060 & 0.060 & 0.060 & 0.060 & 0.060 & 0.060 & 0.060 & 0.060 & 0.060 & 0.060 & 0.060 \\
\hline Antioxidant $^{3}$ & 0.010 & 0.010 & 0.010 & 0.010 & 0.010 & 0.010 & 0.010 & 0.010 & 0.010 & 0.010 & 0.010 & 0.010 \\
\hline Enzyme blend & 0.000 & 0.000 & 0.000 & 0.000 & 0.005 & 0.005 & 0.005 & 0.005 & 0.000 & 0.000 & 0.000 & 0.000 \\
\hline \multicolumn{13}{|l|}{ Calculated composition } \\
\hline Metabolizable energy, kcal/kg & 3.025 & 3.025 & 3.025 & 3.025 & 3.100 & 3.100 & 3.100 & 3.100 & 3.100 & 3.100 & 3.100 & 3.100 \\
\hline Crude protein, \% & 18.8 & 18.8 & 18.8 & 18.8 & 19.15 & 19.15 & 19.15 & 19.15 & 19.15 & 19.15 & 19.15 & 19.15 \\
\hline Digestible lysine, \% & 1.030 & 1.030 & 1.030 & 1.030 & 1.050 & 1.050 & 1.050 & 1.050 & 1.050 & 1.050 & 1.050 & 1.050 \\
\hline Digestible methionine, \% & 0.467 & 0.466 & 0.466 & 0.466 & 0.479 & 0.479 & 0.479 & 0.479 & 0.479 & 0.479 & 0.479 & 0.479 \\
\hline Digestible methionine + cystine,$\%$ & 0.731 & 0.731 & 0.731 & 0.731 & 0.746 & 0.746 & 0.746 & 0.746 & 0.746 & 0.746 & 0.746 & 0.746 \\
\hline Digestible threonine, \% & 0.700 & 0.700 & 0.700 & 0.700 & 0.714 & 0.714 & 0.714 & 0.714 & 0.714 & 0.714 & 0.714 & 0.714 \\
\hline Digestible tryptophan, \% & 0.200 & 0.202 & 0.204 & 0.207 & 0.206 & 0.208 & 0.210 & 0.213 & 0.206 & 0.208 & 0.210 & 0.213 \\
\hline Total glycine+serine, \% & 1.669 & 1.686 & 1.704 & 1.722 & 1.704 & 1.722 & 1.739 & 1.757 & 1.704 & 1.722 & 1.739 & 1.757 \\
\hline Digestible valine, \% & 0.784 & 0.788 & 0.792 & 0.796 & 0.799 & 0.803 & 0.807 & 0.811 & 0.799 & 0.803 & 0.807 & 0.811 \\
\hline Digestible isoleucine, \% & 0.721 & 0.717 & 0.713 & 0.709 & 0.740 & 0.735 & 0.731 & 0.727 & 0.740 & 0.735 & 0.731 & 0.727 \\
\hline Dig. arginine & 1.153 & 1.188 & 1.222 & 1.256 & 1.186 & 1.220 & 1.255 & 1.289 & 1.186 & 1.220 & 1.255 & 1.289 \\
\hline Digestible phenylalanine + tyrosine, \% & 1.442 & 1.445 & 1.448 & 1.451 & 1.471 & 1.474 & 1.477 & 1.480 & 1.471 & 1.474 & 1.477 & 1.480 \\
\hline Digestible histidine, \% & 0.477 & 0.470 & 0.463 & 0.456 & 0.484 & 0.477 & 0.470 & 0.463 & 0.484 & 0.477 & 0.470 & 0.463 \\
\hline Linoleic acid, \% & 1.584 & 2.880 & 3.486 & 4.092 & 1.511 & 3.750 & 4.355 & 4.961 & 1.511 & 3.750 & 4.355 & 4.961 \\
\hline Calcium, \% & 0.740 & 0.740 & 0.740 & 0.740 & 0.820 & 0.820 & 0.820 & 0.820 & 0.820 & 0.820 & 0.820 & 0.820 \\
\hline Available phosphorus, \% & 0.310 & 0.310 & 0.310 & 0.310 & 0.410 & 0.410 & 0.410 & 0.410 & 0.410 & 0.410 & 0.410 & 0.410 \\
\hline Sodium, \% & 0.208 & 0.208 & 0.208 & 0.208 & 0.208 & 0.208 & 0.208 & 0.208 & 0.208 & 0.208 & 0.208 & 0.208 \\
\hline Crude fiber, \% & 2.70 & 4.20 & 5.70 & 7.20 & 2.72 & 4.22 & 5.72 & 7.22 & 2.72 & 4.22 & 5.72 & 7.22 \\
\hline Neutral detergent fiber, \% & 11.81 & 14.26 & 16.71 & 19.16 & 11.58 & 14.03 & 16.49 & 18.94 & 11.58 & 14.03 & 16.49 & 18.94 \\
\hline Acid detergent fiber, $\%$ & 4.69 & 5.91 & 7.13 & 8.35 & 4.68 & 5.90 & 7.12 & 8.34 & 4.68 & 5.90 & 7.12 & 8.34 \\
\hline
\end{tabular}

${ }^{1}$ Vitamin premix (content/kg product): vit. A - 10,000,000 IU; vit. D3 - 2,000,000 IU; vit. E - 30,000 IU; vit. B1 - 2.0 g; vit. B2 - 6.0 g; vit. B6 - 4.0 g; vit. B12 - 0.015 g; pantothenic acid - $12.0 \mathrm{~g}$; biotin - $0.1 \mathrm{~g}$; vit. K3 - $3.0 \mathrm{~g}$; folic acid - $1.0 \mathrm{~g}$; nicotinic acid - $50.0 \mathrm{~g}$; Se - $250.0 \mathrm{mg}$

2 Mineral premix (content/kg product): Fe - $80 \mathrm{~g} ; \mathrm{Cu}-10 \mathrm{~g} ; \mathrm{Co}-2 \mathrm{~g} ; \mathrm{Mn}-80 \mathrm{~g} ; \mathrm{Zn}-50 \mathrm{~g} ; \mathrm{I}-1 \mathrm{~g}$

3 Antioxidant: BHT (Butyl Hydroxy Toluene). 
Table 2 - Nutritional values attributed to the enzyme blend ${ }^{1}$

\begin{tabular}{|c|c|c|}
\hline Nutritional matrix & Per kg & $\begin{array}{l}\text { Added at } \\
\text { levels of }\end{array}$ \\
\hline Metabolizable energy, kcal/kg & 1.500 .000 & 75 \\
\hline Crude protein, \% & 7.000 & 0.350 \\
\hline Digestible lysine, \% & 180 & 0.009 \\
\hline Digestible methionine, \% & 80 & 0.004 \\
\hline Digestible methionine + cystine, \% & 140 & 0.007 \\
\hline Digestible threonine, \% & 120 & 0.006 \\
\hline Digestible tryptophan, \% & 40 & 0.002 \\
\hline Digestible valine, \% & 200 & 0.010 \\
\hline Digestible arginine, \% & 230 & 0.012 \\
\hline \multicolumn{3}{|l|}{ Composition of the enzyme blend (\%) } \\
\hline Endo-1,3(4)-beta-glucanase & \multicolumn{2}{|c|}{14.0} \\
\hline Xylanase & \multicolumn{2}{|c|}{11.0} \\
\hline 6-phytase & \multicolumn{2}{|c|}{5.0} \\
\hline Inert material & \multicolumn{2}{|c|}{70.0} \\
\hline
\end{tabular}

${ }^{1}$ Rovabio Excel AP®

Table 3 - Chemical composition of sunflower meal

\begin{tabular}{ll}
\hline Sunflower meal & \\
Dry matter $^{1}$ & 91.37 \\
\hline Metabolizable energy, kcal/kg² & 1.983 \\
\hline Crude protein, \% $^{1}$ & 25.00 \\
\hline Digestible lysine, \% ${ }^{2}$ & 0.634 \\
\hline Digestible methionine, \% & \\
\hline Digestible methionine + cystine, \% & 0.504 \\
\hline Digestible threonine, \% & 0.858 \\
\hline Digestible tryptophan, \% & 0.765 \\
\hline Total glycine+serine, \% & 0.315 \\
\hline Digestible valine, \% & 2.560 \\
\hline Digestible isoleucine, \% & 1.140 \\
\hline Digestible arginine, \% & 0.970 \\
\hline Digestible phenylalanine + tyrosine, \% & 2.080 \\
\hline Digestible histidine, \% & 2.050 \\
\hline Linoleic acid, \% & 0.570 \\
\hline Ether extract, \% & 0.600 \\
\hline Ashes, \% & 2.120 \\
\hline Calcium, \% & 4.730 \\
\hline Total phosphorus, \% & 0.140 \\
\hline Available phosphorus, \% & 0.939 \\
\hline Sodium, \% & 0.310 \\
\hline Crude fiber, \% & 0.200 \\
\hline Neutral detergent fiber, \% & 22.37 \\
\hline Acid detergent fiber, \% & 45.19 \\
\hline IAnses & 21.35 \\
\hline
\end{tabular}

${ }^{1}$ Analyses performed at the animal nutrition laboratory, UFV.

${ }^{2}$ Average values according to the NRC (1994), FEDNA (2003), INRA (2004) and Tavernari et al. (2010).

Performance and carcass trait results were analyzed using the PROC GLM of SASC statistical package according to a factorial arrangement at $5 \%$ significance level. Linear and quadratic functions were applied to determine the optimal level of sunflower meal, and the test of Student-Newmann-Keul to evaluate the inclusion of the enzyme blend.

\section{RESULTS AND DISCUSSION}

Average temperature recorded during the experiment was $21.5^{\circ} \mathrm{C}\left(16.0\right.$ and $27.0^{\circ} \mathrm{C}$ minimum and maximum temperatures, respectively), which was within the thermal comfort zone for broilers at the evaluated age.

Feed intake was influenced by the interaction between feed formulation and SFM levels $(p<0.05$, Table 4). Higher feed intake was obtained when feeds were calculated with nutritional deficiency and no inclusion of the enzyme blend (NC) than in the feeds calculated with adequate nutritional levels (PC) at the level of $8.0 \%$ inclusion of SFM. The influence of dietary energy content influences feed intake, which may explain this result. According to Nascimento et al. (2005), feed intake is closely related to feed energy level, supporting the results obtained. The inclusion of the enzyme blend did not affect feed intake ( $p>0.05$, Table 4). Abdelrahman \& Saleh (2007) also did not find any influence of the inclusion of glucanase in SFM diets. On the other hand, Raza et al. (2009) verified higher weight gain and better feed conversion ratio when adding carbohydrases to SFM diets fed to broilers.

There was a linear effect $(p<0.05$, Table 4$)$ of sunflower meal levels on feed intake, which increased only in the PC diets (Table 5), differently from the reports of Furlan et al. (2001) and Tavernari et al. (2009), who did not find any significant differences in that parameter up to the levels of 20 and 25\% SFM inclusion, respectively. Abdelrahman \& Saleh (2007) obtained higher feed intake with the inclusion of $10 \%$ sunflower meal.

Despite containing soluble fibers, sunflower meal also contains high insoluble fiber levels, which are represented by the approximate difference between NDF and ADF (45.19 and $21.35 \%$, respectively). Insoluble fibers increase fecal volume and defecation frequency, reducing intestinal transit time (Mattos \& Martins, 2000). Poultry have a short digestive tract, and therefore, high fiber levels that increase passage rate reduce nutrient absorption (Macari, 2008). We hypothesize that a lower nutrient absorption when broilers were fed higher sunflower meal levels could lead to compensatory feed intake.

The interaction between feed formulations and SFM level was not significant for weight gain ( $p>0.05$, Table 4). Weight gain was not affected by the addition of the enzyme blend ( $p>0.05$, Table 4). On the other hand, SFM levels had a negative linear influence $(p<0.05$, Table 4) on weight gain, as shown by the equation 
Table 4 - Performance of 21- to 42-d-old broilers fed diets with increasing sunflower meal levels and supplemented or not with an enzyme blend.

\begin{tabular}{|c|c|c|c|c|c|}
\hline & \multicolumn{5}{|c|}{ Sunflower meal levels } \\
\hline & $0 \%$ & $8 \%$ & $16 \%$ & $24 \%$ & \\
\hline & \multicolumn{4}{|c|}{ Feed intake (g/bird) } & Mean \\
\hline NC & $4.068 a$ & $4.117 a$ & $3.943 a$ & $4.029 a$ & 4.039 \\
\hline $\mathrm{NC}+\mathrm{EB}$ & $4.008 a$ & $4.015 a b$ & $3935 a$ & $4.068 a$ & 4.007 \\
\hline PC & $3.881 \mathrm{~b}$ & $3.920 b$ & $4.002 \mathrm{a}$ & $3.601 a$ & 3.851 \\
\hline Mean & 3.986 & 4.017 & 3.960 & 3.899 & \\
\hline ANOVA & $\mathrm{FF}^{\mathrm{a}}=0.0094^{*}$ & $\mathrm{SFM}^{\beta}=0.1235^{\mathrm{ns}}$ & FF X & & $C V(\%)=3.28$ \\
\hline \multirow[t]{2}{*}{ Probability } & - & NS & & & \\
\hline & \multicolumn{4}{|c|}{ Weight gain (g/bird) } & Mean \\
\hline NC & 2.183 & 2.128 & 2.074 & 2.022 & 2.102 \\
\hline $\mathrm{NC}+\mathrm{EB}$ & 2.142 & 2.158 & 2.041 & 2.067 & 2.102 \\
\hline PC & 2.167 & 2.124 & 2.081 & 2.063 & 2.109 \\
\hline Mean & 2.164 & 2.137 & 2.065 & 2.051 & \\
\hline ANOVA & - & $\mathrm{SFM}=<.0001^{\star}$ & $F F \times S$ & $7^{\text {ns* }}$ & $C V(\%)=2.99$ \\
\hline \multirow[t]{2}{*}{ Probability } & - & L & & & \\
\hline & \multicolumn{4}{|c|}{ Feed conversion ratio $(\mathrm{g} / \mathrm{g})$} & Mean \\
\hline NC & $1.863 a$ & $1.935 a$ & $1.901 a$ & $1.993 a$ & 1.923 \\
\hline $\mathrm{NC}+\mathrm{EB}$ & $1.871 \mathrm{a}$ & $1.861 \mathrm{ab}$ & $1.928 a$ & $1.968 a$ & 1.907 \\
\hline PC & $1.791 b$ & $1.846 b$ & $1.923 a$ & $1.746 a$ & 1.826 \\
\hline Mean & 1.842 & 1.880 & 1.917 & 1.902 & \\
\hline ANOVA & Trat $=<0.0001 *$ & $\mathrm{SFM}=<0.0001 *$ & \multicolumn{2}{|c|}{$\mathrm{FF} X \mathrm{SFM} \gamma=0.0026^{*}$} & $C V(\%)=2.88$ \\
\hline Probability & - & L & \multicolumn{2}{|c|}{ L } & \\
\hline
\end{tabular}

${ }^{a} \mathrm{NC}=$ negative control; $\mathrm{NC}+\mathrm{EB}=$ negative control + enzyme blend; $\mathrm{PC}=$ positive control.

${ }^{\beta}$ SFM $=$ sunflower meal percentage in the feed.

$\checkmark$ Interaction between feed formulation and SFM.

${ }^{a, b}$ Means followed by different letters in the same column are statistically different by the SNK test $(p<0.05)$

${ }^{n s}$ Not significant by the $F$ test $(p>0.05)$.

* Significant by the $F$ test $(p>0.05)$.

$Q$ - quadratic effect $(p \leq 0.05)$ of sunflower meal.

$L-$ linear effect $(p \leq 0.05)$ of sunflower meal.

NS - not significant.

in Table 5. The recommendations of sunflower meal dietary inclusion in the present study are different from those of other authors, who reported that inclusion of $12.04 \%, 12.0 \%, 10.0 \%$, and up to $25 \%$ did not affect weight gain (Furlan et al., 2001; Pinheiro et al., 2002; Abdelrahman \& Saleh, 2007; Tavernari et al., 2009, respectively).

Modern broiler strains present increasing nutritional requirements, and demand highly digestible diets with increasing nutritional values. Sunflower meal has high fiber content (NDF $=45.19 \%$ ), which negatively affects nutrient absorption, resulting in worse performance.

The interaction of feed formulation with SFM levels also influenced $(p<0.05$, Table 4) feed conversion ratio, which was worse when broilers were fed the NC diets compared with the PC diets for the SFM level of $0.8 \%$.
Table 5 - Regression equations of weight gain and feed conversion ratio of broilers fed diets with increasing sunflower meal levels and supplemented or not with an enzyme blend.

\begin{tabular}{|c|c|}
\hline Feed formulation ${ }^{a}$ & Feed intake (g/bird) \\
\hline NC & Not significant $(p>0.05)$ \\
\hline $\mathrm{NC}+\mathrm{EB}$ & Not significant $(p>0.05)$ \\
\hline \multirow[t]{3}{*}{ PC } & Feed intake $=3.878 .3+6.725$ SFM $^{\beta}\left(R^{2} 0.97\right)$ \\
\hline & Weight gain (g/bird) \\
\hline & Weight gain $=2.165 .9-5.1021 \mathrm{SFM}(\%)\left(R^{2} 0.83\right)$ \\
\hline Feed formulation & Feed conversion ratio (g/g) \\
\hline NC & feed conversion ratio $=1.8696+0.0028$ SFM $\left(R^{2} 0.48\right)$ \\
\hline $\mathrm{NC}+\mathrm{EB}$ & Feed conversion ratio $=1.8776+0.0034$ SFM $\left(R^{2} 0.83\right)$ \\
\hline PC & Feed conversion ratio $=1.7974+0.0055$ SFM $\left(R^{2} 0.92\right)$ \\
\hline
\end{tabular}


Sunflower meal levels had a linear effect $(p<0.05$, Table 5) on feed conversion ratio. However, different equations were obtained for each feed formulation (NC, NC+ EB, and PC). The equations show that feed conversion ratio worsened as sunflower meal level increased. At a same SFM inclusion level, feed formulation worsened feed conversion ratio in the following order: NC, NC+EB, and PC. These results do not agree with the findings of several authors (Furlan et al., 2001; Pinheiro et al., 2002; Abdelrahman \& Saleh, 2007; Tavernari et al., 2009).

The best production efficiency index (PEI) was obtained in broilers fed the PC diet with not inclusion of sunflower meal (Table 6). However, the best economic efficiency index (EEI) was obtained in the broilers fed the NC+EB diet containing 8.0\% sunflower meal (Table 6). Therefore, despite resulting in worse performance, the inclusion of $8.0 \%$ sunflower meal may be economically viable, as well as the addition of the enzyme blend to this feed. On the other hand, Furlan et al. (2001) and Tavernari et al. (2009) verified better EEI when sunflower meal was not included in the feeds, but Pinheiro et al. (2002) reported that the inclusion of $4.0 \%$ SFM promoted the best EEl.

Table 6 - Production efficiency index (PEI) and economic efficiency index (EEI) of broilers fed diets with increasing sunflower meal levels and supplemented or not with an enzyme blend.

\begin{tabular}{|c|c|c|c|c|c|}
\hline \multicolumn{6}{|c|}{ Sunflower meal levels } \\
\hline & $0 \%$ & $8 \%$ & $16 \%$ & $24 \%$ & \\
\hline \multicolumn{5}{|c|}{ Production efficiency index } & Mean \\
\hline$N C^{a}$ & 522.88 & 492.38 & 499.76 & 464.55 & 494.89 \\
\hline $\mathrm{NC}+\mathrm{EB}$ & 516.19 & 521.59 & 487.48 & 476.03 & 500.32 \\
\hline PC & 550.39 & 524.48 & 492.48 & 485.35 & 513.17 \\
\hline Mean & 529.82 & 512.82 & 493.24 & 475.31 & \\
\hline \multicolumn{5}{|c|}{ Economic efficiency index (\%) } & Mean \\
\hline NC & 99.26 & 95.31 & 98.64 & 93.46 & 96.67 \\
\hline $\mathrm{NC}+\mathrm{EB}$ & 99.33 & 100.00 & 97.31 & 94.29 & 97.73 \\
\hline PC & 99.37 & 96.16 & 91.72 & 91.05 & 94.58 \\
\hline Mean & 99.32 & 97.16 & 95.89 & 92.93 & \\
\hline
\end{tabular}

a $\mathrm{NC}=$ negative control; $\mathrm{NC}+\mathrm{EB}=$ negative control + enzyme blend; $\mathrm{PC}=$ positive control.

There was no effect of feed formulation (NC, $\mathrm{NC}+\mathrm{EB}$, or $\mathrm{PC}$ ) or of the interaction between feed formulation and sunflower meal levels ( $p>0.05)$ on carcass traits (Tables 7 and 8). Sunflower meal levels linearly affected carcass, breast, breast fillet, and abdominal fat weights ( $p<0.05$, Tables 7 and 8 ). It was concluded the increasing sunflower meal levels reduce some carcass traits, as described by the equations in Table 9.

Table 7 - Carcass weight, breast weight, and breast yield of broilers fed diets with increasing sunflower meal levels and supplemented or not with an enzyme blend.

\begin{tabular}{|c|c|c|c|c|c|}
\hline \multicolumn{6}{|c|}{ Sunflower meal levels } \\
\hline & $0 \%$ & $8 \%$ & $16 \%$ & $24 \%$ & \\
\hline & \multicolumn{4}{|c|}{ Carcass weight (g/bird) } & Mean \\
\hline NC & 2.116 & 2.098 & 1.972 & 1.943 & 2.032 \\
\hline $\mathrm{NC}+\mathrm{EB}$ & 2.084 & 2.097 & 1.994 & 1.947 & 2.030 \\
\hline PC & 2.066 & 2.018 & 2.007 & 1.974 & 2.016 \\
\hline Mean & 2.089 & 2.071 & 1.991 & 1.955 & \\
\hline ANOVA & $\begin{array}{c}\mathrm{FF}^{a}= \\
0.6169^{\text {ns }}\end{array}$ & $\begin{array}{c}\mathrm{SFM}^{\beta}= \\
<0.0001^{*}\end{array}$ & FF X SFM & $=0.1473^{\text {ns }}$ & $\begin{array}{c}C V(\%)= \\
3.45\end{array}$ \\
\hline \multirow[t]{2}{*}{ Probability } & - & L & & & \\
\hline & \multicolumn{4}{|c|}{ Breast weight (g/bird) } & Mean \\
\hline NC & 769 & 747 & 700 & 679 & 724 \\
\hline $\mathrm{NC}+\mathrm{EB}$ & 743 & 736 & 706 & 711 & 724 \\
\hline PC & 735 & 709 & 714 & 690 & 712 \\
\hline Mean & 749 & 731 & 707 & 693 & \\
\hline ANOVA & $\begin{array}{c}F F= \\
0.3748^{\text {ns }}\end{array}$ & $\begin{array}{c}\mathrm{SFM}= \\
<0.0001 \text { * }\end{array}$ & FF X SFM & $=0.2288^{\mathrm{ns}}$ & $\begin{array}{c}C V(\%)= \\
5.47\end{array}$ \\
\hline \multirow[t]{2}{*}{ Probability } & - & L & & & \\
\hline & \multicolumn{4}{|c|}{ Breast yield (\%) } & Mean \\
\hline NC & 36.32 & 35.65 & 35.50 & 34.93 & 35.600 \\
\hline $\mathrm{NC}+\mathrm{EB}$ & 35.64 & 35.11 & 35.40 & 36.55 & 35.675 \\
\hline PC & 35.55 & 35.12 & 35.55 & 34.90 & 35.280 \\
\hline Mean & 35.837 & 35.293 & 35.483 & 35.460 & \\
\hline ANOVA & $\begin{array}{c}F F= \\
0.5145^{\text {ns }}\end{array}$ & $\begin{array}{c}S F M= \\
0.6112^{\text {ns }}\end{array}$ & FF X SFM & $0.2753^{\mathrm{ns}}$ & $\begin{array}{c}C V(\%)= \\
4.04\end{array}$ \\
\hline Probability & - & NS & & & \\
\hline
\end{tabular}

${ }^{a} \mathrm{NC}=$ negative control; $\mathrm{NC}+\mathrm{EB}=$ negative control + enzyme blend; $\mathrm{PC}=$ positive control.

${ }^{\beta}$ SFM $=$ sunflower meal percentage in the feed.

$\checkmark$ Interaction between feed formulation and SFM

${ }^{n s}$ Not significant by the $F$ test $(p>0.05)$.

* Significant by the $\mathrm{F}$ test $(p>0.05)$.

$Q$ - quadratic effect $(p \leq 0.05)$ of sunflower meal.

$L-$ linear effect $(p \leq 0.05)$ of sunflower meal.

NS - not significant.

Carcass results reflect the reduced weight gain as a function of increasing SFM levels in the feed. These results are different from those reported by Oliveira 
et al. (2003) and Tavernari et al. (2009), who did not find any influence on carcass traits of SFM levels up to $25.0 \%$ and $30.0 \%$, respectively, as well as no significant differences in weight gain, which explains carcass results.

Table 8 - Breast fillet weight, and breast fillet, thighs and drumstick, and abdominal fat yields of broilers fed diets with increasing sunflower meal levels and supplemented or not with an enzyme blend.

\begin{tabular}{|c|c|c|c|c|c|}
\hline \multicolumn{6}{|c|}{ Sunflower meal levels } \\
\hline & $0 \%$ & $8 \%$ & $16 \%$ & $24 \%$ & \\
\hline \multicolumn{5}{|c|}{ Breast fillet weight (g/bird) } & Mean \\
\hline NC & 594 & 589 & 541 & 522 & 561 \\
\hline$N C+E B$ & 575 & 551 & 543 & 550 & 555 \\
\hline PC & 583 & 545 & 544 & 533 & 551 \\
\hline Mean & 584 & 562 & 543 & 535 & \\
\hline ANOVA & $\begin{array}{c}F F^{a}= \\
0.5023^{n s}\end{array}$ & $\begin{array}{c}\mathrm{SFM}^{\beta}= \\
<0.0001^{*}\end{array}$ & \multicolumn{2}{|c|}{$\mathrm{FF} X \mathrm{SFM}^{\mathrm{r}}=0.1472^{\mathrm{ns}}$} & $C V(\%)=6.20$ \\
\hline \multirow[t]{2}{*}{ Probability } & - & L & \multicolumn{2}{|l|}{ NS } & \\
\hline & \multicolumn{4}{|c|}{ Breast fillet yield (\%) } & Mean \\
\hline NC & 28.05 & 28.08 & 27.48 & 26.82 & 27.61 \\
\hline $\mathrm{NC}+\mathrm{EB}$ & 27.60 & 26.32 & 27.21 & 28.24 & 27.34 \\
\hline PC & 28.22 & 27.02 & 27.12 & 26.97 & 27.33 \\
\hline Mean & 27.96 & 27.14 & 27.27 & 27.34 & \\
\hline ANOVA & $\begin{array}{c}F F= \\
0.2154^{\text {ns }}\end{array}$ & $\begin{array}{c}\text { SFM }= \\
0.2154^{\text {ns }}\end{array}$ & $\mathrm{FF} \times \mathrm{SFM}=$ & $0.1050^{\text {ns }}$ & $C V(\%)=5.26$ \\
\hline \multirow[t]{2}{*}{ Probability } & - & NS & NS & & \\
\hline & \multicolumn{4}{|c|}{ Thighs and drumstick weight (g/bird) } & Mean \\
\hline NC & 590 & 593 & 580 & 561 & 581 \\
\hline$N C+E B$ & 600 & 578 & 589 & 575 & 585 \\
\hline PC & 585 & 587 & 571 & 571 & 578 \\
\hline Mean & 591 & 586 & 580 & 569 & \\
\hline ANOVA & $\begin{array}{c}F F= \\
0.6529^{\text {ns }}\end{array}$ & $\begin{array}{c}\text { SFM = } \\
0.0745^{\text {ns }}\end{array}$ & $\mathrm{FF} \times \mathrm{SFM}=$ & $0.7422^{\mathrm{ns}}$ & $C V(\%)=5.19$ \\
\hline \multirow[t]{2}{*}{ Probability } & - & NS & NS & & \\
\hline & \multicolumn{4}{|c|}{ Abdominal fat (g/bird) } & Mean \\
\hline NC & 34 & 41 & 30 & 27 & 33 \\
\hline$N C+E B$ & 35 & 36 & 33 & 34 & 34 \\
\hline PC & 36 & 37 & 33 & 33 & 35 \\
\hline Mean & 35 & 38 & 32 & 31 & \\
\hline ANOVA & $\begin{array}{c}F F= \\
0.5420^{\text {ns }}\end{array}$ & $\begin{array}{c}\text { SFM }= \\
0.0047^{*}\end{array}$ & $\mathrm{FF} \times \mathrm{SFM}=$ & $0.3242^{\text {ns }}$ & $C V(\%)=20.99$ \\
\hline Probability & - & L & NS & & \\
\hline
\end{tabular}

a $\mathrm{NC}=$ negative control; $\mathrm{NC}+\mathrm{EB}=$ negative control + enzyme blend; $\mathrm{PC}=$ positive control.

${ }^{\beta}$ SFM $=$ sunflower meal percentage in the feed.

$\mathrm{v}$ Interaction between feed formulation and SFM.

${ }^{n s}$ Not significant by the $F$ test ( $\left.p>0.05\right)$.

* Significant by the $\mathrm{F}$ test ( $p>0.05)$.

$Q$ - quadratic effect $(p \leq 0.05)$ of sunflower meal.

$L$ - linear effect $(p \leq 0.05)$ of sunflower meal.

NS - not significant.
Table 9 - Regression equations of the parameters carcass weight, breast weight, breast fillet weight, and abdominal fat of broilers fed diets with increasing sunflower meal levels and supplemented or not with an enzyme blend.

Carcass weight $(\mathrm{g} / \mathrm{bird})=2.098 .6-6.0312 \mathrm{SFM}^{1}\left(\mathrm{R}^{2} 0.94\right)$

Breast weight $(\mathrm{g} / \mathrm{bird})=748.6-2.3926$ SFM $\left(R^{2} 0.99\right)$

Breast fillet weight $(\mathrm{g} / \mathrm{bird})=580.81-2.0846 \mathrm{SFM}\left(\mathrm{R}^{2} 0.96\right)$

Abdominal fat $(\mathrm{g} / \mathrm{bird})=36.625-0.2161 \mathrm{SFM}\left(\mathrm{R}^{2} 0.97\right)$

${ }^{1} \mathrm{SFM}=$ sunflower meal percentage in the feed.

\section{CONCLUSIONS}

Increasing dietary addition of SFM reduced weight gain and worsened feed conversion ratio $(P>0.05)$. The best EEl was obtained with the $\mathrm{NC}+\mathrm{EB}$ diet containing $8.0 \%$ SFM. The inclusion of sunflower meal in broiler feeds negatively influenced performance and carcass parameters. The dietary inclusion of the enzyme blend did not improve the evaluated parameters. However, the inclusion of $8 \%$ SFM and EB addition to the diet improves EEI.

\section{ACKNOWLEDGMENTS}

The authors thank Fundação de Amparo à Pesquisa do Estado de Minas Gerais for their support.

\section{REFERENCES}

Abdelrahman MM, Saleh FH. Performance of Broiler Chickens Fed on CornSunflower Meal Diets with B-Glucanase Enzyme. Jordan Journal of Agricultural Sciences 2007;3(3):272-280.

Bellaver C, Fialho, ET, Protas JFS, et al. Radícula de malte na alimentação de suínos em crescimento e terminação. Pesquisa Agropecuária Brasileira 1985;20(8):969-974.

Fundacíon Española para el Desarrollo de la Nutrición Animal. Tablas de composición y valor nutritivo de alimentos para la fabricación de piensos compuestos. $2^{a}$ ed. Madrid: Mundi-prensa; 2003. 423p.

Furlan AC, Mantovani C, Murakami AE, et al. Utilização do farelo de girassol na alimentação de frangos de corte. Revista Brasileira de Zootecnia 2001;30(1):158-164.

Institute National de loa Recherche Agronomique. Tablas de composición y de valor nutritivo de las materias primas destinadas a los animales de interés ganadero: cerdos, aves, bovinos, ovinos, caprinos, conejos, caballos, peces. Madrid: Ediciones Mundi Prensa; 2004. p.194-197.

Kocher A, Choct M, Porter MD, et al. The effects of enzyme addition to broiler diets containing high concentrations of canola or sunflower meal. Poultry Science 2000;79: 1767-1774.

Macari M, Furlan, RL, Gonzales E. Fisiologia aviária aplicada a frangos de corte. Jaboticabal: FUNEP/UNESP; 2008. 375p.

Mattos LL, Martins IS. Consumo de fibras alimentares em população adulta. Revista de Saúde Pública 2000;34(1):50-55. 
Nascimento AH, Gomes PC, Rostagno HS. et al. Valores de energia metabolizável de farinhas de penas e de vísceras determinados com diferentes níveis de inclusão e duas idades das aves. Revista Brasileira de Zootecnia 2005;34(3):877-881.

National Research Council. Nutrient requirements of poultry. $9^{\text {th }}$ ed. Washington, D.C.: National Academy of Sciences; 1994. 155p.

Oliveira MC, Martins FF, Almeida CV, et al. Efeito da inclusão de bagaço de girassol na ração sobre o desempenho e rendimento de carcaça de frangos de corte. Revista Portuguesa de Zootecnia 2003;10(2):107116.

Pinheiro JW, Fonseca NAN, Silva CA, et al. Farelo de girassol na alimentação de frangos de corte em diferentes fases de desenvolvimento. Revista Brasileira de Zootecnia 2002;31(supl 3):1418-1425.

Porto WS, Carvalho CGP, Pinto RJB, et al. Evaluation of sunflowers cultivars for central Brazil. Scientia Agrícola 2008;65(2):139-144.

Raza S, Ashraf M, Pasha TN, et al. Effect of enzyme supplementation of broiler diets containing varying level of sunflower meal and crude fiber. Pakistan Journal of Botany 2009;41(5):2543-2550.
Rosa PM, Antoniassi R, et al. Chemical composition of Brazilian sunflower varieties. Helia 2009;32(50):145-155

Rostagno HS, Albino LFT, Donzele JL, et al. Tabelas brasileiras para aves e suínos: composição de alimentos e exigências nutricionais. Viçosa, MG: Universidade Federal de Viçosa; 2005. 168 p.

SAS Program. User guide for personal computer. Cary: SAS Institute; 2002 $846 \mathrm{p}$.

Tavernari FC, Albino LFT, Morata RL, et al. Inclusion of sunflower meal, with or without enzyme supplementation, in broiler diets. Brazilian Journal of Poultry Science 2008;10 (4):233-238.

Tavernari FC, Dutra Junior WM, Albino LFT, et al. Efeito da utilização de farelo de girassol na ração sobre o desempenho de frangos de corte. Revista Brasileira de Zootecnia 2009;38(9):1745-1750.

Tavernari FC, Morata RL, Ribeiro Jr V, et al. Avaliação nutricional e energética do farelo de girassol para aves. Arquivos Brasileiros de Medicina Veterinária e Zootecnia 2010;62(1):172-177 